

\title{
Dosimetric Comparison between Varian Halcyon Analytical Anisotropic Algorithm and Acuros XB Algorithm for Planning of RapidArc Radiotherapy of Cervical Carcinoma
}

\author{
Jonathan Mbewe ${ }^{\circledR}$, Sakhele Shiba \\ Division of Medical Physics, Groote Schuur Hospital, Cape Town, South Africa
}

Received 30 November 2021

Revised 10 December 2021

Accepted 13 December 2021

Corresponding author

Jonathan Mbewe

(Jonathan.Mbewe@uct.ac.za)

Tel: 27-21-404-6266

Fax: 27-21-404-3260
Purpose: The Halcyon radiotherapy platform at Groote Schuur Hospital was delivered with a factory-configured analytical anisotropic algorithm (AAA) beam model for dose calculation. In a recent system upgrade, the Acuros $X B(A X B)$ algorithm was installed. Both algorithms adopt fundamentally different approaches to dose calculation. This study aimed to compare the dose distributions of cervical carcinoma RapidArc plans calculated using both algorithms.

Methods: A total of 15 plans previously calculated using the AAA were retrieved and recalculated using the $\mathrm{AXB}$ algorithm. Comparisons were performed using the planning target volume (PTV) maximum (max) and minimum (min) doses, D95\%, D98\%, D50\%, D2\%, homogeneity index (HI), and conformity index $(\mathrm{Cl})$. The mean and max doses and D2\% were compared for the bladder, bowel, and femoral heads.

Results: The AAA calculated slightly higher targets, D98\%, D95\%, D50\%, and $\mathrm{Cl}$, than the AXB algorithm (44.49 Gy vs. $44.32 \mathrm{~Gy}, P=0.129 ; 44.87 \mathrm{~Gy}$ vs. $44.70 \mathrm{~Gy}, P=0.089 ; 46.00 \mathrm{~Gy}$ vs. 45.98 Gy, $P=0.154$; and 0.51 vs. $0.50, P=0.200$, respectively). For target min dose, $D 2 \%$, max dose, and $\mathrm{HI}$, the AAA scored lower than the AXB algorithm (41.24 Gy vs. $41.30 \mathrm{~Gy}, P=0.902 ; 47.34 \mathrm{~Gy}$ vs. $47.75 \mathrm{~Gy}, P<0.001 ; 48.62 \mathrm{~Gy}$ vs. $50.14 \mathrm{~Gy}, P<0.001$; and 0.06 vs. $0.07, P=0.002$, respectively). For bladder, bowel, and left and right femurs, the AAA calculated higher mean and max doses.

Conclusions: Statistically significant differences were observed for PTV D2\%, max dose, $\mathrm{HI}$, and bowel max dose $(P>0.05)$.

Keywords: Halcyon, Acuros XB, Analytical anisotropic algorithm, RapidArc, Radiotherapy

\section{Introduction}

Cervical cancer $(\mathrm{CaCx})$ is the fourth most prevalent cancer affecting women. Low- and middle-income countries account for the highest morbidity burden, with $>85 \%$ of deaths in 2018 [1]. In South Africa, at least 7,000 new disease cases were diagnosed in 2012 [2] at different stages of presentation. Generally, external beam radiotherapy (EBRT) with curative intent is indicated in approximately $60 \%$ of all patients with $\mathrm{CaCx}$ [3]. At the Division of Radiation Oncology, Groote Schuur Hospital, curative EBRT for $\mathrm{CaCx}$ is delivered with image-guided volumetric modulated arc therapy (VMAT) on the Halcyon platform (Varian Medical Systems, Palo Alto, CA, USA).

VMAT is an advanced form of intensity-modulated radiotherapy (IMRT). Besides leaf motion in multileaf collima- 
tors, the dose rate and gantry rotation speeds are modulated and synchronized to achieve dose distributions that are at least comparable with those of IMRT in significantly less treatment time [4]. It relies on inverse treatment planning methods to create optimized dose distributions. Provided systematic and random uncertainties are managed well, the steep dose gradients achievable with VMAT may maximize the target dose and suppress the dose to organs at risk, thereby improving the therapeutic ratio of this technique over classical EBRT methods. Random uncertainties associated with inaccurate patient positioning are reduced on Halcyon by enforcing the acquisition of pretreatment $\mathrm{kV}$ cone-beam computed tomography (CT) for position verification and correction at every treatment session. To minimize systematic errors associated with incorrect modeling of the radiation beam, Halcyon is delivered with a hardcoded, factory-configured beam model and dose calculation engine, which the end-user must validate before clinical implementation [5]. At our center, this validation was performed independently according to the recommendations of the Medical Physics Practice Guideline 5.a. of the American Association of Physicists in Medicine (AAPM) [6].

The Eclipse treatment planning system (Varian Medical Systems) on the Halcyon platform was initially configured with the analytical anisotropic algorithm (AAA) radiation dose calculation engine as the sole option. In 2020, the Acuros XB (AXB) algorithm was released commercially as the second purchasable option. Although both algorithms share the same multiple-source model for primary photons, extrafocal photons, and electron contamination [7], the crucial difference lies in modeling radiation transport and dose deposition. The AAA uses the convolution/superposition method, for which dose deposition kernels are precomputed using Monte Carlo in water. Tissue is assumed to be materially composed of water, and CT-number to electrondensity calibration curves must be derived to account for tissue heterogeneity. To improve the dose calculation accuracy, the kernels are scaled in the forward and lateral directions in proportion to the electron density encountered in the voxels. The calculated dose is reported as dose to water. The AXB algorithm transports radiation and calculates dose deposition by solving the linear Boltzmann transport equation (LBTE), akin to Monte Carlo methods, but employing deterministic, grid-based Boltzmann solvers instead of random sampling [8]. The LBTE is a group of integrodifferential equations that describe the transport and interaction of radiation in matter by assuming that the incident radiation interacts only with matter [9]. The AXB algorithm requires interaction cross sections of material elements in the voxels to model fluence transport and dose deposition in matter. For this purpose, the treatment planning system provides a material table containing material type, mass density, and interaction cross section for biological materials (adipose tissue, lung, muscle, cartilage, and bone) and 16 nonbiological materials. The AXB algorithm supports a maximum (max) Hounsfield unit (HU) of 8,000. Materials with higher HU have material manually allocated by the user. The AXB algorithm inherently reports the dose calculation as dose to material. The closest rivals to the AXB algorithm are Monte Carlo codes, which hitherto have been the gold standard in radiotherapy dose calculations. However, their clinical adoption has not been widespread because calculation times are typically too long for practical applications. The few Monte Carlo codes approved for clinical implementation compromise accuracy for calculation speed and are not generally considered true Monte Carlo codes.

Some researchers have reported the accuracy of the AXB algorithm for dose computation to be on par with that of Monte Carlo, with the advantage of increased calculation speed [10]. In clinical radiotherapy, dose calculation accuracy may affect treatment outcomes of tumor control and toxicities. Because the target volumes and organs at risk in $\mathrm{CaCx}$ radiotherapy are in areas of high tissue heterogeneity, accurate modeling of the dose distribution is particularly important to understand the probability of observing complications and the desired therapeutic outcomes. Although some studies have performed dosimetric comparisons between the AAA and AXB algorithm for other treatment sites and platforms [11], to the best of our knowledge, no such comparison exists for VMAT of $\mathrm{CaCx}$ on the Halcyon platform. Such comparisons would be necessary because the beam models delivered with this system are not user adjustable. Thus, dosimetric comparisons performed on other systems may not necessarily hold for Halcyon. This study considers several dosimetric parameters obtained from $\mathrm{CaCx}$ treatment plans calculated with the AAA and $\mathrm{AXB}$ 
algorithm engines. The study aims to answer the following question: is there a significant difference in the dosimetric performance of the AAA and AXB algorithm?

\section{Materials and Methods}

This was a retrospective and nonrandomized study. We retrieved 15 Halcyon RapidArc treatment plans created in Eclipse v15.6 for radiotherapy of $\mathrm{CaCx}$ from the hospital's Aria Oncology Information System (Varian Medical System). The method of VMAT implemented by Varian Medical Systems is referred to as RapidArc. These plans had been previously calculated using the AAA and approved for treatment. Structure sets were designed following local protocols [12], which required the tumor clinical target volume (CTV-T) to include the gross tumor volume, cervix,

Table 1. The average PTV dose-volume parameters from dose distributions calculated using AAA and AXB

\begin{tabular}{lrrr}
\hline \multicolumn{1}{c}{ PTV } & \multicolumn{1}{c}{ AAA } & AXB & $P$-value \\
\hline Mean (Gy) & $46.00 \pm 0.00$ & $46.00 \pm 0.00$ & $<0.001$ \\
D95\% (Gy) & $44.87 \pm 0.24$ & $44.70 \pm 0.26$ & 0.089 \\
D98\% (Gy) & $44.49 \pm 0.30$ & $44.32 \pm 0.28$ & 0.129 \\
D50\% (Gy) & $46.00 \pm 0.04$ & $45.98 \pm 0.04$ & 0.154 \\
D2\% (Gy) & $47.34 \pm 0.15$ & $47.75 \pm 0.16$ & $<0.001$ \\
Max (Gy) & $48.62 \pm 0.35$ & $50.14 \pm 0.40$ & $<0.001$ \\
Min (Gy) & $41.24 \pm 1.40$ & $41.30 \pm 0.97$ & 0.902 \\
HI & $0.06 \pm 0.01$ & $0.07 \pm 0.01$ & 0.002 \\
CI & $0.51 \pm 0.02$ & $0.50 \pm 0.02$ & 0.200 \\
MU & $726.24 \pm 82.41$ & $722.19 \pm 89.18$ & 0.959 \\
\hline
\end{tabular}

Values are presented as mean \pm standard deviation.

PTV, planning target volume; AAA, analytical anisotropic algorithm; AXB, Acuros XB; Max, maximum; Min, minimum; HI, homogeneity index; $\mathrm{CI}$, conformity index; $\mathrm{MU}$, monitor unit. uterus, parametrium, and vagina. The nodal CTV (CTV$\mathrm{N}$ ) had been created to include the inguinal and obturator nodes. The final tumor planning target volume (PTV-T) had been created by adding a margin of $15 \mathrm{~mm}$ in the superior, anterior, and inferior directions and $10 \mathrm{~mm}$ in the posterior direction. The nodal PTV (PTV-N) had been generated by adding a 7-mm margin symmetrically around the CTV-N. Contours of the organs at risk included left and right femoral heads, bladder, bowel bag, and bone marrow. All plans had a treatment prescription of $46 \mathrm{~Gy}$ in 20 fractions.

Copies of the original plans were made, and threedimensional dose distributions were recalculated using the Halcyon AXB algorithm. The original normalization to the PTV mean dose, calculation grid size of $2.5 \mathrm{~mm}$, and the inherent dose to material reporting were used. The dosimetric comparison was performed by assessing the PTV coverage metrics of D95\%, D98\%, D50\%, D2\%, homogeneity index (HI), and conformity index (CI). For the organs at risk, differences were assessed by considering the mean dose, max dose, and D2\% of the left and right femoral heads, bladder, and bowel bag. The normal tissue integral dose (NTID) predicted by both algorithms was also considered. The definitions of NTID [13], CI, and HI adopted in this study are shown in equations (1), (2), and (3), respectively:

NTID=mean dose $\times$ volume of normal tissue outside PTV (1)

$$
C I=\frac{\text { Volume of } 100 \% \text { isodose surface }}{\text { Volume of PTV }}
$$

$$
\mathrm{HI}=\frac{D 2 \%-D 98 \%}{D 50 \%}
$$

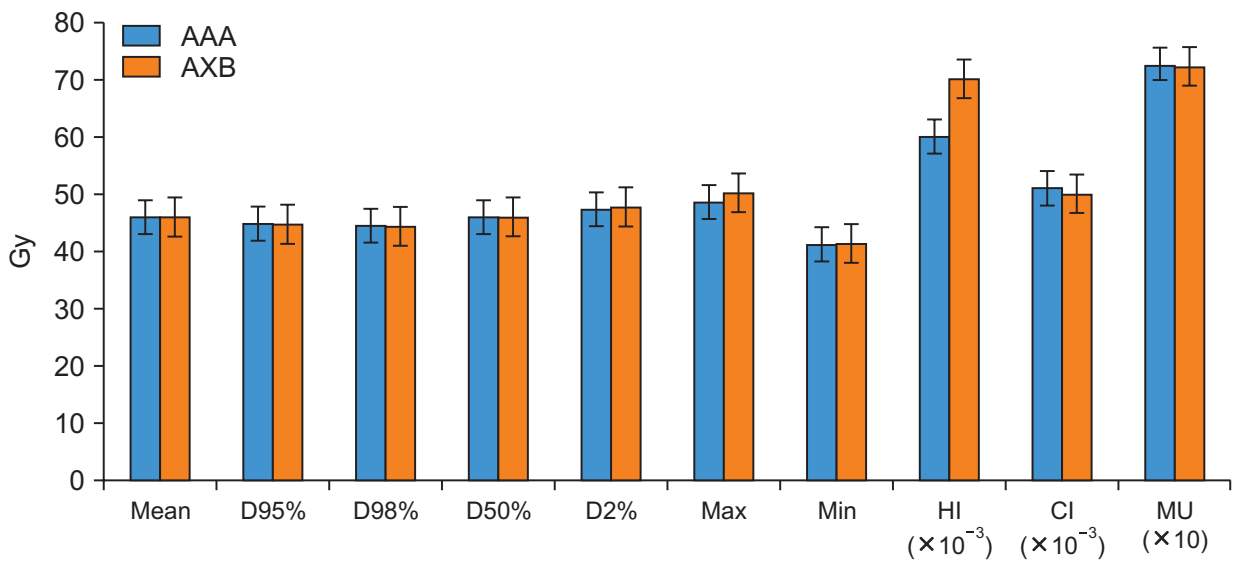

Fig. 1. Comparison of dosimetric parameters for PTV coverage calculated using AAA and AXB. PTV, planning target volume; AAA, analytical anisotropic algorithm; AXB, Acuros XB; Max, maximum; Min, minimum; HI, homogeneity index; $\mathrm{CI}$, conformity index; MU, monitor unit. 
In practice, the NTID was derived in the treatment planning system by subtracting the PTV-T and PTV-N structures from the body structure and assigning the difference as a separate structure. This structure's mean dose in Gy and volume in liters were then obtained from the dose-volume histogram.

Statistical comparison was performed using a two-tailed t-test with a significance level set at $0.05 ; P$-values $<0.05$ were considered statistically significant with a $95 \%$ confidence limit. Statistical analyses of the data were performed using a web-based statistics calculator [14].

\section{Results}

Table 1 and Fig. 1 summarize the PTV dosimetric parameters extracted from the RapidArc plans whose dose distributions were calculated using the AAA and AXB algorithm. The data show similar target coverage across most metrics, except for D2\%, max dose, and HI, which yielded 47.34 Gy vs. $47.75 \mathrm{~Gy}, 48.62$ Gy vs. $50.14 \mathrm{~Gy}$, and 0.06 vs. 0.07 $(P<0.05)$ for AAA vs. AXB algorithm, respectively. The AAA metrics for PTV were slightly higher, except for D2\% (47.34 Gy vs. 47.75 Gy, $P<0.001$ ), max dose (48.62 Gy vs. 50.14 Gy, $P<0.001$ ), and min dose (41.24 Gy vs. $41.30 \mathrm{~Gy}, P=0.902)$, as well as HI (0.06 vs. $0.07, P=0.002)$. Table 2 and Fig. 2 summarize the dosimetric parameters relating to organs at risk. The AAA calculates slightly higher metrics than the AXB algorithm, although the differences in most cases are not statistically significant. The sole exception is the bowel max dose, which yielded 48.07 Gy vs. $49.18 \mathrm{~Gy}(P<0.001)$ for AAA vs. AXB algorithm. Furthermore, no significant differences were observed in the average monitor unit (MU) (726.24 vs. 722.19) and NTID (both $3.52 \times 10^{2} \mathrm{~L} \cdot \mathrm{Gy}$ ). This result shows that treatment delivery with a plan calculated using one algorithm would not be more time efficient than the other. No reduction was observed in the size of the low-dose bath predicted using either algorithm. Fig. 3 shows the comparison of graphical dose distributions calculated using the two algorithms. Fig. 4 and 5 show comparisons of dose-volume histograms of PTV and organs at risk, respectively.

Table 2. The average organs at risk dose-volume parameters from dose distributions calculated using AAA and AXB

\begin{tabular}{lccc}
\hline Structure & AAA (Gy) & AXB (Gy) & $P$-value \\
\hline Bladder & & & \\
Mean & $42.18 \pm 2.80$ & $42.10 \pm 2.84$ & 0.939 \\
Max & $47.50 \pm 1.16$ & $47.80 \pm 1.06$ & 0.468 \\
D2\% & $46.53 \pm 1.47$ & $46.55 \pm 1.49$ & 0.978 \\
Bowel & & & \\
Mean & $21.50 \pm 3.02$ & $21.41 \pm 3.00$ & 0.939 \\
Max & $48.07 \pm 0.28$ & $49.18 \pm 0.41$ & $<0.001$ \\
D2\% & $46.10 \pm 1.49$ & $46.25 \pm 1.54$ & 0.783 \\
Rt femur & & & \\
Mean & $17.16 \pm 3.02$ & $16.98 \pm 2.95$ & 0.871 \\
Max & $44.10 \pm 0.86$ & $43.82 \pm 1.01$ & 0.615 \\
D2\% & $38.73 \pm 1.47$ & $38.14 \pm 1.45$ & 0.422 \\
Lt femur & & & \\
Mean & $17.37 \pm 2.70$ & $17.19 \pm 2.70$ & 0.858 \\
Max & $44.12 \pm 2.17$ & $43.99 \pm 2.45$ & 0.882 \\
D2\% & $38.98 \pm 2.13$ & $38.40 \pm 2.10$ & 0.511 \\
NTID $\left(\times 10^{2} \mathrm{~L} \cdot G y\right)$ & $3.52 \pm 0.653$ & $3.52 \pm 0.668$ & 0.987
\end{tabular}

AAA, analytical anisotropic algorithm; AXB, Acuros XB; Max, maximum; Rt, right; Lt, Left; NTID, normal tissue integral dose.

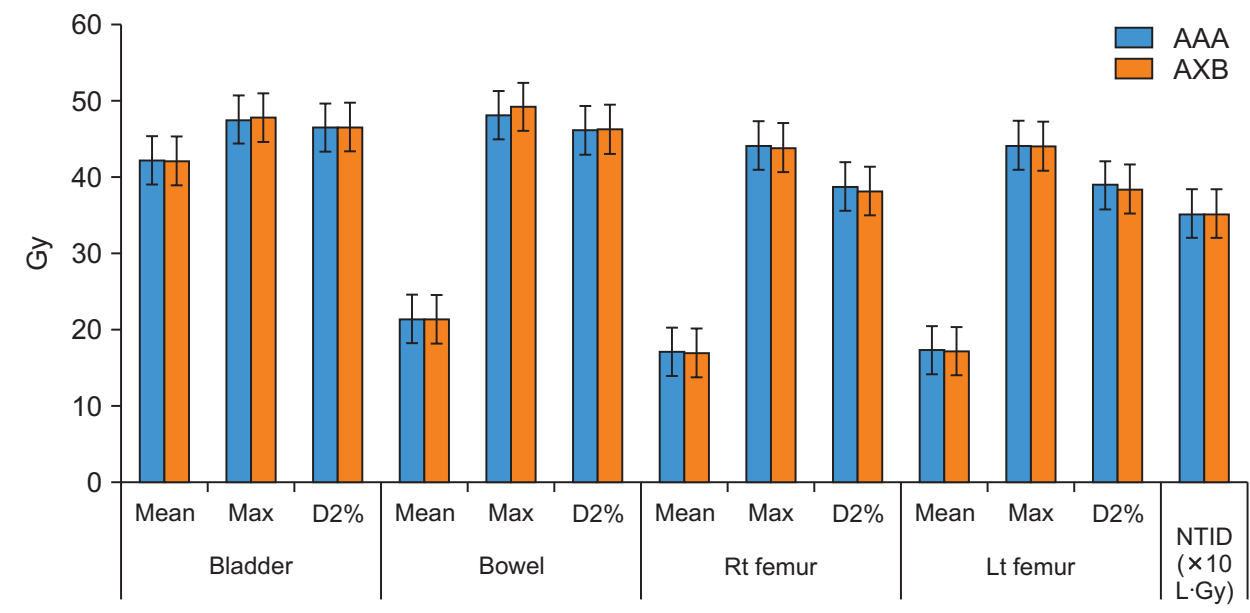

Fig. 2. Comparison of dosimetric parameters for organs at risk calculated using AAA and AXB. AAA, analytical anisotropic algorithm; AXB, Acuros XB; Rt, right; Lt, Left; NTID, normal tissue integral dose; Max, maximum. 


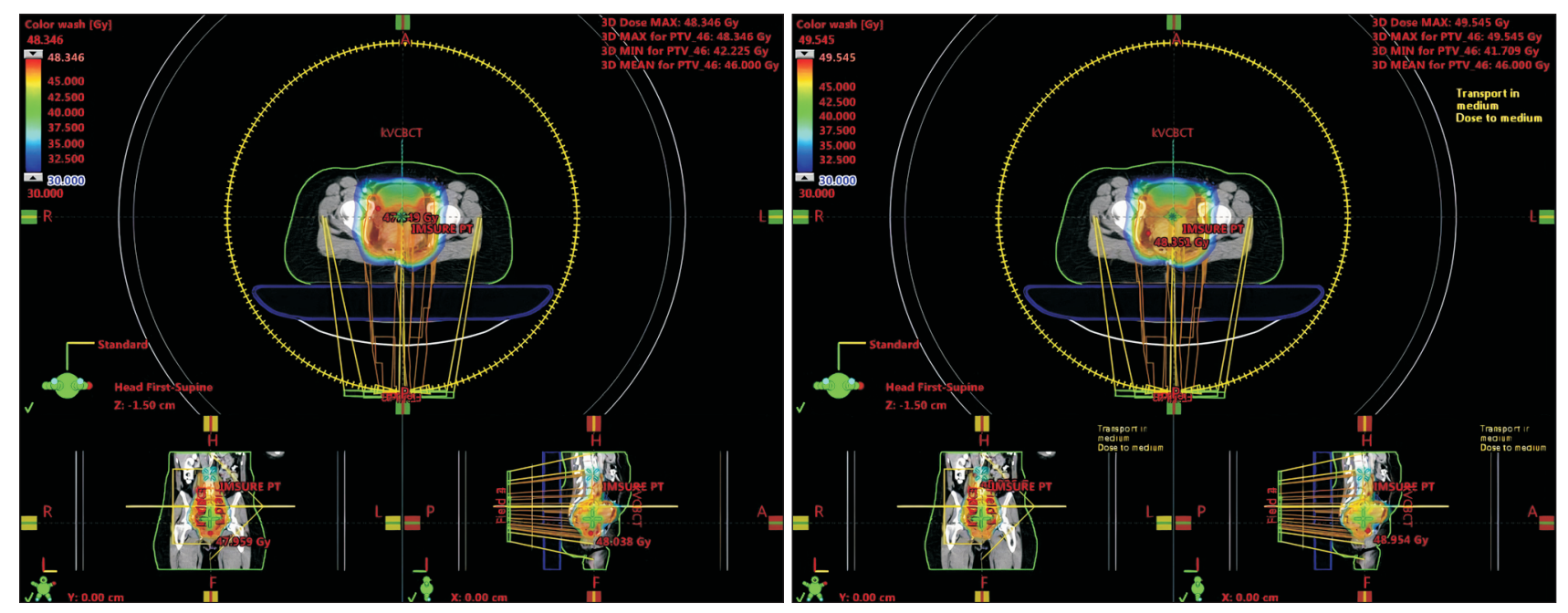

Fig. 3. Comparison of dose distributions calculated using AAA and AXB on the left- and right-hand sides, respectively, for a selected treatment plan. AAA, analytical anisotropic algorithm; AXB, Acuros XB.

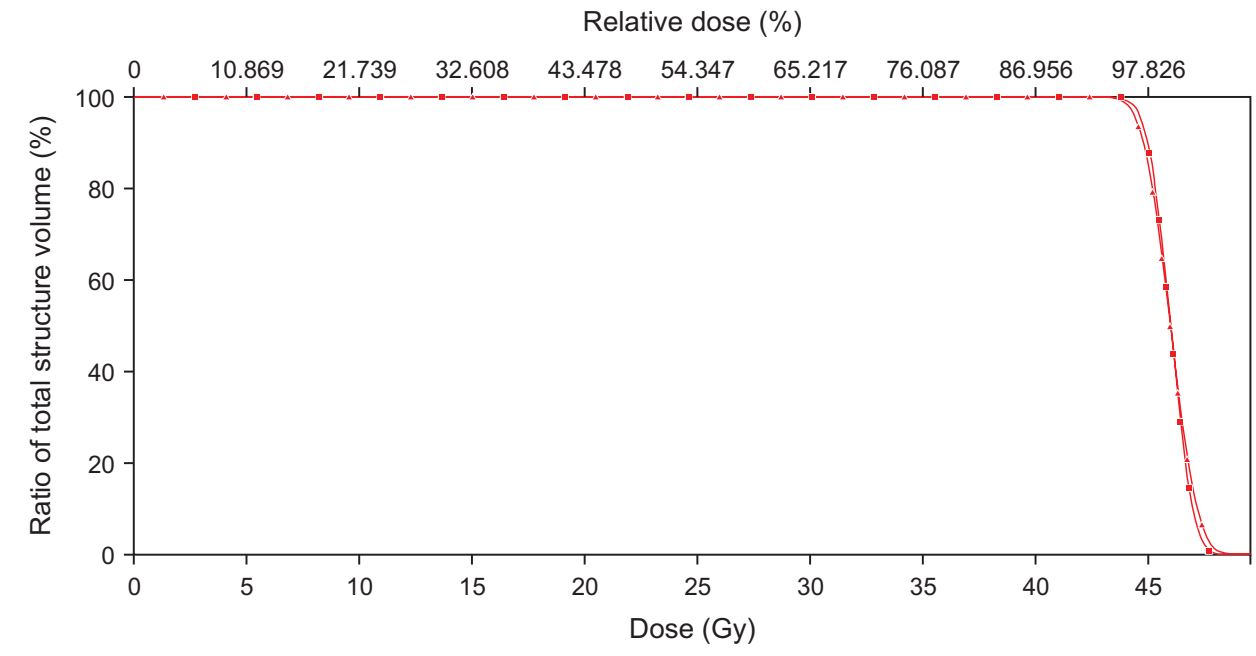

Fig. 4. PTV dose-volume histograms calculated using AAA (squares) and AXB (triangles) for a selected treatment plan.

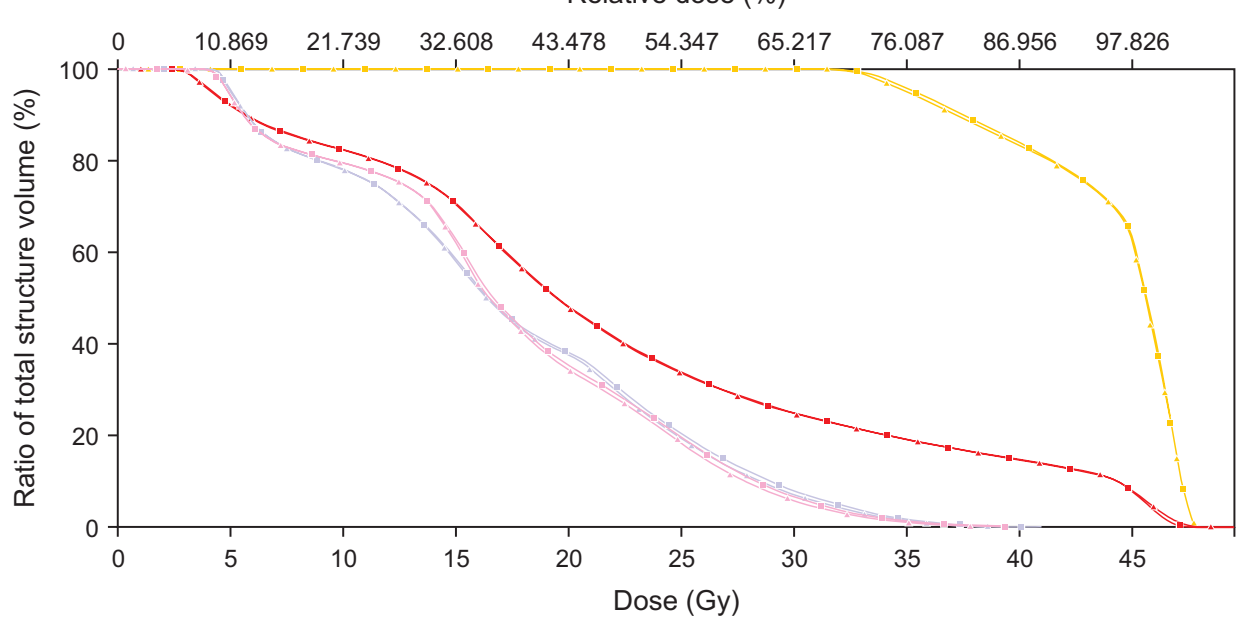

Fig. 5. Organs at risk dose-volume histograms calculated using AAA (squares) and AXB (triangles) for a selected treatment plan. The left femur, right femur, bowel, and bladder histograms are in purple, blue, red, and yellow, respectively. 


\section{Discussion}

This study performed a dosimetric comparison of $\mathrm{CaCx}$ RapidArc plans whose dose distributions were calculated using the AAA and AXB algorithm. The comparison used quantitative metrics and qualitative tools to consider PTV dose coverage and sparing of organs at risk. Because the treatment target area is usually in an environment of tissue homogeneity, it is customary to expect significant differences in the dose distributions modeled by the AAA and AXB algorithm. This study reported statistically significant differences in PTV D2\%, max dose, and HI $(P<0.05)$, for which the AXB algorithm predicted higher values. AAA predicted a higher D95\% than AXB, but the difference was not statistically significant. Differences in the organs at risk dose metrics were found to be not significant $(P>0.05)$, except for the bowel max dose, for which the AXB algorithm predicted a higher value than AAA $(P<0.001)$. These findings are inconsistent with those of the study by Kumar et al. [11], who conducted a similar study, albeit on a C-arm, TrueBeam STx (Varian Medical Systems) linear accelerator, using a prescription of $50.4 \mathrm{~Gy}$ in 28 fractions. They reported no significant differences in PTV D95\% and statistically significant differences in PTV mean dose (52.73 Gy vs. 52.87 Gy), D2\% (54.88 Gy vs. 55.32 Gy), max dose (57.29 Gy vs. 52.26 Gy), min dose (43.93 Gy vs. 43.04 Gy), HI (0.100 vs. 0.108$)$, CI (1.000 vs. 1.006), and NTID (11.78 vs. 11.72). Of the organs at risk dose metrics, all were found to have significant differences except for the mean rectum dose $(P=0.681)$. However, that study adopted a treatment plan normalization of $100 \%$ prescription dose to $95 \%$ PTV, whereas a normalization to target mean dose was used in this study. This fact could certainly result in different conclusions regarding the mean PTV dose at least and probably the other dose-volume metrics as well. Additionally, even though it is not mentioned, beam modeling of Varian Medical System's C-arm linear accelerators is a task performed by the user, who may tune the model to their preference. However, Halcyon's beam model is factory configured and the user does not have the option for tuning.

A more reasonable comparison would be with other Halcyon systems, with the final dose calculations reported as dose-to-material, such as AXB algorithm dose. For the
AAA, $1 \%$ upward scaling of the absolute dose to water calibration is recommended for this purpose by the report of AAPM Task Group 329 [15]. The same report advises against changing the AXB algorithm's dose reporting from dose-tomaterial reporting to dose-to-water. AXB inherently reports dose as dose-to-water. Unfortunately, to the best of our knowledge, similar studies could not be found in the literature. It is hoped that as more Halcyon users commission the AXB algorithm, dosimetric evaluations identical to this one will be conducted.

\section{Conclusions}

A dosimetric plan comparison between Halcyon AAA and AXB algorithm was conducted for RapidArc CaCx plans. Statistically significant differences were found for PTV D2\%, max dose, HI, and bowel max dose. However, the differences may not necessarily be clinically significant. Thus, either algorithm may perform calculations of $\mathrm{CaCx}$ RapidArc with comparable accuracy.

\section{Conflicts of Interest}

The authors have nothing to disclose.

\section{Availability of Data and Materials}

All relevant data are within the paper and its Supporting Information files.

\section{Author Contributions}

Conceptualization: Jonathan Mbewe. Data curation: Jonathan Mbewe and Sakhele Shiba. Formal analysis: Jonathan Mbewe. Funding acquisition: none. Investigation: Jonathan Mbewe and Sakhele Shiba. Methodology: Jonathan Mbewe. Project administration: Jonathan Mbewe. Resources: Jonathan Mbewe. Software: Jonathan Mbewe and Sakhele Shiba. Supervision: Jonathan Mbewe. Validation: Jonathan Mbewe and Sakhele Shiba. Writing-original draft: Jonathan Mbewe. Writing_-review and editing: Sakhele Shiba. 


\section{References}

1. Arbyn M, Weiderpass E, Bruni L, de Sanjosé S, Saraiya M, Ferlay J, et al. Estimates of incidence and mortality of cervical cancer in 2018: a worldwide analysis. Lancet Glob Health. 2020;8:e191-e203.

2. Jordaan S, Michelow P, Richter K, Simoens C, Bogers J. A review of cervical cancer in South Africa: previous, current and future. Health Care Curr Rev. 2016;4:1000180.

3. Delaney G, Jacob S, Barton M. Estimation of an optimal radiotherapy utilization rate for gynecologic carcinoma: part I--malignancies of the cervix, ovary, vagina and vulva. Cancer. 2004;101:671-681.

4. Otto K. Volumetric modulated arc therapy: IMRT in a single gantry arc. Med Phys. 2008;35:310-317.

5. Nguyen D, Pietri FJ, Sporea C, Khodri M. Patient Quality assurance of the new halcyon linear accelerator (Varian). Phys Med. 2018;56(Suppl 1):10-11.

6. Smilowitz JB, Das IJ, Feygelman V, Fraass BA, Kry SF, Marshall IR, et al. AAPM medical physics practice guideline 5 . a.: commissioning and QA of treatment planning dose calculations- megavoltage photon and electron beams. J Appl Clin Med Phys. 2015;16:14-34.

7. Sievinen J, Ulmer W, Kaissl W. AAA photon dose calculation model in Eclipse ${ }^{\mathrm{TM}}$. Crowley: Varian Medical Systems; 2005.

8. Vassiliev ON, Wareing TA, McGhee J, Failla G, Salehpour MR, Mourtada F. Validation of a new grid-based Boltzmann equation solver for dose calculation in radiotherapy with photon beams. Phys Med Biol. 2010;55:581-598.

9. Akpochafor MO, Aweda MA, Durosinmi-Etti FA, Adeneye SO, Omojola AD. Simulation of the Linear Boltzmann Transport Equation in modelling of photon beam data. IOSR J Appl Phys. 2014;5:72-86.

10. Bedford JL. Calculation of absorbed dose in radiotherapy by solution of the linear Boltzmann transport equations. Phys Med Biol. 2019;64:02TR01.

11. Kumar L, Kishore V, Bhushan M, Kumar P, Chaudhary RL. Dosimetric impact of Acuros XB on cervix radiotherapy using RapidArc technique: a dosimetric study. Rep Pract Oncol Radiother. 2021;26:582-589.

12. Bhagaloo V, Bhim N, Hunter A, Fakie N. A dosimetric comparison of volumetric modulated arc therapy with threedimensional conformal radiotherapy in the treatment of cervical cancer. South Afr J Oncol. 2021;5:a149.

13. Aoyama H, Westerly DC, Mackie TR, Olivera GH, Bentzen SM, Patel RR, et al. Integral radiation dose to normal structures with conformal external beam radiation. Int J Radiat Oncol Biol Phys. 2006;64:962-967.

14. Social Science Statistics. T-test calculator for 2 independent means. Social Science Statistics, [cited 2021 Jul 22]. Available from: https://www.socscistatistics.com/tests/ studentttest/default2.aspx.

15. Kry SF, Feygelman V, Balter P, Knöös T, Charlie Ma CM, Snyder M, et al. AAPM task group 329: reference dose specification for dose calculations: dose-to-water or doseto-muscle? Med Phys. 2020;47:e52-e64. 